\title{
A DFT study of the effects of Sc doping on electronic and optical properties of CdS nanoparticles
}

\author{
Shafiq Ur Rehman ${ }^{1}$, Abdul Majid $^{2 *}$, NAJMAl HASSAN ${ }^{1}$, AbDul ShaKoor $^{3}$, G. Murtaza ${ }^{4}$, \\ SALAH-DIN KHAN ${ }^{5}$ \\ ${ }^{1}$ Physics Department, Hazara University Mansehra, Pakistan \\ ${ }^{2}$ Physics Department, University of Gujrat, Gujrat, Pakistan \\ ${ }^{3}$ Physics Department, Hazara University Mansehra, Pakistan \\ ${ }^{4}$ Physics Department, Islamia College Peshawar, Pakistan \\ ${ }^{5}$ Sustainable Energy Technologies Center, College of Engineering, King Saud University, \\ PO Box 300, Riyadh 11421, Saudi Arabia
}

\begin{abstract}
In the present work a systematic study was carried out to understand the influence of Sc doping on electronic and optical properties of CdS nanoparticles. The geometry optimization and symmetry computation for CdS and Sc doped CdS nanoparticles using Density Functional Theory (DFT) on B3LYP level with the QZ4P for Cd and DZ2P for sulphur and Sc were performed by Amsterdam Density Functional (ADF). The results show that HOMO-LUMO gap as well as electronic and optical properties of CdS clusters vary with Sc doping. The HOMO-LUMO gap is affected by the dopant and its value decreases to $0.6 \mathrm{eV}$. Through considering the numerical integration scheme in the ADF package, we investigated different vibrational modes and our calculated Raman and IR spectra are consistent with the reported result. The calculated IR and Raman peaks of CdS and Sc doped CdS clusters were in the range of 100 to $289 \mathrm{~cm}^{-1}, 60 \mathrm{~cm}^{-1}$ to $350 \mathrm{~cm}^{-1}$ and $99 \mathrm{~cm}^{-1}$ to $282 \mathrm{~cm}^{-1}, 60 \mathrm{~cm}^{-1}$ to $350 \mathrm{~cm}^{-1}$, respectively, which was also confirmed by experiment as well as a blue shift occurrence. Subsequently, for deeper research of pure and doped CdS clusters, their absorption spectra were calculated using time-dependent DFT method.
\end{abstract}

Keywords: Sc doped CdS; vibrational modes; Raman; IR; absorption spectrum; H-L gap, DOS, isosurface plots, Density Functional Theory

(C) Wroclaw University of Technology.

\section{Introduction}

Nanomaterials have attracted great research and industrial interest in recent years because of their unique chemical and physical properties [1,2]. The studies of semiconductor nanoparticles have been an interesting field of research for more than two decades. This is because they give an opportunity to understand the physical properties in low dimensions and to explore their vast potential for application in, e.g., optoelectronics [3]. Over the past two decades, investigations on quantum sized semiconductor particles have increased enormously due to their novel applications in electronic and photonic devices [4-7]. The functionality of these devices has strong correlation with the electronic and structural properties of the material used. These

*E-mail: najamalhassan@yahoo.com properties depend upon the vibrational characteristics of the materials which are, in turn, related to the size of nanoparticles and can be best explored using infrared and Raman scattering techniques [8-10]. II - VI semiconductor nanoparticles have proven to fulfill this requirement to large extent. The research on II - VI semiconductor nanoparticles have shown that these materials are much better than III - V semiconductors especially in the field of solar cells. In addition, they are more exciting because of their relatively wide direct band gap, causing absorption or emission of light in the visible spectrum, i.e. $\mathrm{ZnO}, \mathrm{CdS}$ and $\mathrm{HgSe}$ are materials having a wide range of applications $[11,12]$ in the field of optoelectronics, solar cells, photoresistors, biological signal amplification, lasers, etc.

Cadmium sulphide (CdS) is a compound semiconductor of direct band gap $(\sim 2.4 \mathrm{eV})$ that 
is suitable for optoelectronics application and biological labels [13-17]. In 1993, the high quality quantum dots of $\mathrm{CdS}$ were synthesized for the first time. They emitted different colors depending upon their size and band gap. Currently, modification of $\mathrm{CdS}$ particles by dopant incorporation has become a hot topic. Doping with proper transition metal elements via introducing acceptor or donor level in host nanocrystals is an effective approach to tune the materials properties [18]. Transition metal (TM) (Mn, Fe, Co, etc) doped $\mathrm{CdS}$ has drawn considerable attention as it offers a great opportunity to integrate electrical, optical and magnetic properties into a single material, which makes it an ideal candidate for non volatile memory, magneto-optical and future spintronics devices [19, 20]. The first principle DFT calculations have proven that the system Co-doped CdS (dilutely cobalt-doped cadmium sulphide), in view of the well known application worthy properties of CdS and the potential possibility of its conversion to a diluted magnetic semiconductor, is of interest to spintronics [21]. Similarly, copper impurity changes the type of semiconductor from $n$ to $\mathrm{p}$ type [22]. The $\mathrm{Cu}$ doped $\mathrm{CdS}$ thin films have many applications, such as high efficiency photovoltaic cells and light emitters [23, 24]. Moreover, the doping of copper changes the band gap energy of $\mathrm{CdS}$ and also improves its photoelectric properties [25]. Recently, a few reports have been published on the properties of $\mathrm{Cu}$ doped $\mathrm{CdS}$ layers, for example the study of $\mathrm{Cu}$ doped $\mathrm{CdS}$ homojunction and its rectifying properties [26]. The Fe doped $\mathrm{CdS}$ nanoparticles have shown that impurity atoms increase the fluorescence efficiency [27]. The Mn doped CdS nanoparticles have been used in optoelectronics [28]. It is evident from the literature that the optical properties of $\mathrm{CdS}$ nanoparticles are size dependent. However, when the size becomes smaller than $6 \mathrm{~nm}$, there is a considerable disagreement in the experimental literature, therefore, it is advantageous to use computational methods which are capable of simulating nanoparticles of any size. Of all established computational methods, Density Functional Theory (DFT) is famous for maintaining a perfect balance between computational accuracy and efficiency. It is widely used to study different aspects of molecular systems, i.e. structural, vibrational frequencies and electronic transition for species ranging from nuclei, atoms, molecules to solids. In the present work electronic and optical properties of Sc doped CdS cluster have been calculated using DFT.

\section{Computational details}

Hohenberg and Kohn received in 1998 the Noble Prize for simplifying the many body problem by introducing DFT. Instead of electron wave function they used density functional to solve the problem [29]. The theory gives the exact ground state solution to the structural, electronic, optical and magnetic properties. In present work KohanSham equation [30] was used to calculate the electronic and optical properties of Sc doped $\mathrm{CdS}$ nanoparticles.

All the calculations performed in this work were DFT based and used the ADF program under the approximation GGA-B3LYP. The required basis sets were QZ4P for Cd and DZ2P for both $\mathrm{S}$ and Sc. In present work, ADF molecule was used to analyze HOMO-LUMO gap, DOS, isosurfaces plots, IR spectrum, Raman and excitation spectra of pure and doped CdS nanoparticles. The spectra were displayed and analyzed in the ADF graphical user interface (GUI). The computationally prepared structures for $\mathrm{CdS}$ and doped CdS cluster, shown in Fig. 1, were relaxed by geometry optimization to attain a stable structure with minimized energy. In the calculations we used the core shell and valence shell for $\mathrm{Cd}$ : $1 s^{2} 2 s^{2} 2 p^{6} 3 s^{2} 3 p^{6} 4 s^{2} 3 d^{10} 4 p^{6}$ and $5 s^{2}, 4 d^{10}$. Similarly, for $S: 1 s^{2} 2 s^{2} 2 p^{6}$ and $1 s^{2}, 2 s^{2}, 2 p^{6}, 3 s^{2}, 3 p^{6}$ and for Sc: $1 s^{2}, 2 s^{2}, 2 p^{6}, 3 s^{2}, 3 p^{6}$ core states and $4 s^{2}, 3 d^{1}$ valence states, respectively. In order to carry out a systematic study of the optical and electronic properties of Sc doped $\mathrm{CdS}$ nanoparticles their wurtzite structures in dihedral symmetry were prepared computationally.

We performed geometry optimization of the molecule with energy convergence criteria of $10^{-4}$ Hartree. The physical parameters were obtained after geometry optimization in the output file 

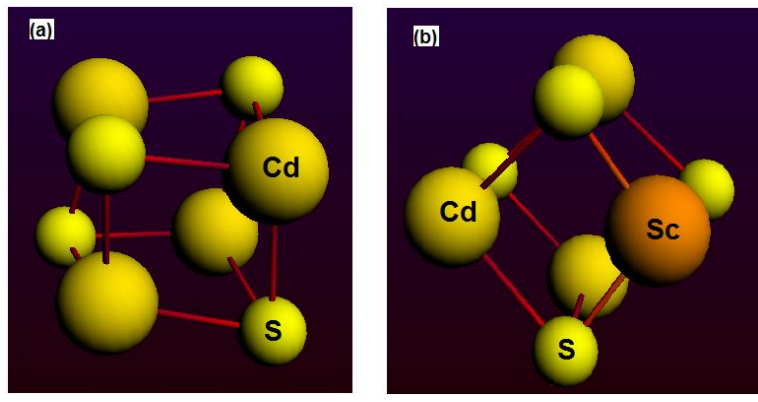

Fig. 1. CdS cluster obtained using DFT based ADF GUI, consisting of (a) pure CdS (b) doped CdS.

consisting of spatial coordinates, charges and atomic mass for all the 8 atoms which form the supercell separately. These physical parameter for pure and doped $\mathrm{CdS}$ cluster are given in Table 1 and Table 2, respectively.

The corresponding bond length and bond angles are shown in Table 3.

\section{Results and discussion}

\subsection{Electronic properties}

According to the molecular orbital theory [31], atomic orbitals (subshells) combine and form a molecular orbital which may have lower or higher energy, known as bonding and anti-bonding molecular orbital. The interactions between these atomic orbitals comprise a particular molecular orbital manifested very proficiently in the molecular orbital structure (MOT). In this work, the active atomic orbitals $5 \mathrm{~s}^{2} 4 \mathrm{~d}^{10}$ and $3 \mathrm{~s}^{2} 3 \mathrm{p}^{4}$ for $\mathrm{Cd}$ and $\mathrm{S}$, respectively, mainly contribute to forming spectroscopically important molecular orbitals (HOMO and LUMO) that participate in the transition eagerly. In contrast, for the doped CdS there is a little contribution of the dopant to LUMO and almost no contribution to the HOMO. The dopant also contributes slightly with its $3 \mathrm{~d}^{1}$ electrons to the core electrons of CdS. HOMO and LUMO are also known as frontier MO which are usually responsible for optical transitions. The MOT structures for $\mathrm{CdS}$ and doped $\mathrm{CdS}$ clusters are given in Fig. 2. The black, blue and grey lines represent the completely filled, partially filled and empty molecular orbitals, respectively. The red lines in the MOT structure show the strength of contribution of each atomic orbital in forming a specific molecular orbital. In the side box, the enlarged view gives the HOMO and LUMO locations and gap between them. Additionally, isosurfaces plots of resulting HOMO and LUMO are given to manifest the contribution of a particular atomic orbital in their formation. We are using conventional abbreviations for bonding molecular orbital (BMO), antibonding molecular orbital (ABMO), atomic orbital (AO), molecular orbital (MO), lowest unoccupied molecular orbital (LUMO), highest occupied molecular orbitals (HOMO) in following sections.

The molecular orbital (MO) structure for CdS in the ground state is given in Fig. 2 and the corresponding HOMO-LUMO are shown in enlarged view boxes. The lower energy active AO's $4 \mathrm{~d}^{10}$ and $3 \mathrm{~s}^{2}$ of $\mathrm{Cd}$ and $\mathrm{S}$, respectively, combine to form filled restricted bonding $\sigma$ and anti-bonding $\sigma^{*}$ MO's. The restricting trait accounts for two electrons with opposite spins $(\uparrow \downarrow)$ that can be accommodated in the same molecular orbital, according to Pauli exclusion principle. The relatively higher energy atomic orbitals $5 \mathrm{~s}^{2}$ and $3 \mathrm{p}^{4}$ of $\mathrm{Cd}$ and $\mathrm{S}$ contribute to forming two bonding $(\sigma, \pi)$ and two antibonding $\left(\sigma^{*}, \pi^{*}\right)$ molecular orbitals. The bonding molecular orbital always has lower energy than the anti-bonding molecular orbital depicting its stabilization. Taking half of the difference of the number of bonding and anti-bonding electrons gives the bond order $(6-0 / 2=3)$ that satisfies $\mathrm{CdS}$, which is triply bonded with one $\sigma$ and two $\pi$ molecular orbitals.

The spectroscopically important MOs HOMO and LUMO, are highlighted in the separate colored box in Fig. 3. The HOMO, pictured in red and black combination, located at $-5.263645 \mathrm{eV}$, contains two electrons with oppositely oriented spins (up and down) in the same state, following the Pauli exclusion principle. There is a single dark red line directed to HOMO from $3 \mathrm{p}^{4}$ atomic orbital of sulphur indicating that $\mathrm{S}$ mainly contributes in HOMO formation. As it is apparent in Fig. 3, the isosurface plots of HOMO show that MO lobes are attracted to the $\mathrm{S}$ end of the CdS cluster. This trend also supports the fact that $S$ is more electronegative than 
Table 1. Physical parameters for the pure CdS cluster in ADF.

\begin{tabular}{ccccccc}
\hline Atoms & \multicolumn{2}{c}{ Spatial extensions in 3D $(\AA)$} & Charge & Charge & Atomic \\
& $\mathrm{X}$ & $\mathrm{Y}$ & $\mathrm{Z}$ & Nucleus & Core & mass $(\mathrm{amu})$ \\
\hline \hline $1 \mathrm{~S}$ & 1.4598 & 1.4598 & 1.4598 & 16.00 & 6.00 & 31.9721 \\
$2 \mathrm{Cd}$ & -1.1323 & 1.1323 & 1.1323 & 48.00 & 48.00 & 113.903 \\
$3 \mathrm{~S}$ & -1.4598 & -1.4598 & 1.4598 & 16.00 & 6.00 & 31.972 \\
$4 \mathrm{Cd}$ & 1.1323 & -1.1323 & 1.1323 & 48.00 & 48.00 & 113.903 \\
$5 \mathrm{Cd}$ & 1.1323 & 1.1323 & -1.1323 & 48.00 & 48.00 & 113.903 \\
$6 \mathrm{~S}$ & -1.4598 & 1.4598 & -1.4598 & 16.00 & 6.00 & 31.9721 \\
$7 \mathrm{Cd}$ & -1.1323 & -1.1323 & -1.1323 & 48.00 & 48.00 & 113.903 \\
$8 \mathrm{~S}$ & 1.4598 & -1.4598 & -1.4598 & 16.00 & 6.00 & 31.9721 \\
\hline
\end{tabular}

Table 2. Physical parameters for a doped CdS cluster in ADF GUI.

\begin{tabular}{ccccccc}
\hline Atoms & \multicolumn{2}{c}{ Spatial coordinate in $3 \mathrm{D}(\AA)$} & Charge & Atomic & mass \\
& $\mathrm{X}$ & $\mathrm{Y}$ & $\mathrm{Z}$ & nucleus & core & (a.m.u $)$ \\
\hline \hline $1 \mathrm{~S}$ & -1.223 & 1.9439 & -1.962 & 16.00 & 6.00 & 31.9721 \\
$2 \mathrm{Cd}$ & -2.064 & 0.000 & -3.678 & 48.00 & 48.00 & 113.9034 \\
$3 \mathrm{~S}$ & -1.1223 & 1.9439 & -1.962 & 16.00 & 6.00 & 31.9721 \\
$4 \mathrm{Sc}$ & 0.0000 & 0.000 & -1.071 & 21.00 & 11.0 & 44.9559 \\
$5 \mathrm{Cd}$ & 1.0307 & 1.7852 & -3.678 & 48.00 & 113.9 & 31.9721 \\
$6 \mathrm{~S}$ & 0.0000 & 0.0000 & -5.339 & 16.00 & 6.00 & 31.9721 \\
$7 \mathrm{Cd}$ & 1.0307 & -1.7852 & -3.678 & 48.00 & 48.00 & 113.9034 \\
$8 \mathrm{~S}$ & 2.2446 & 0.0000 & -1.962 & 16.00 & 6.00 & 31.9721 \\
\hline
\end{tabular}

$\mathrm{Cd}$, thus, it attracts larger part of electrons in $\mathrm{MO}$ with partial positive charge on metallic cadmium and partial negative charge on nonmetallic sulphur.

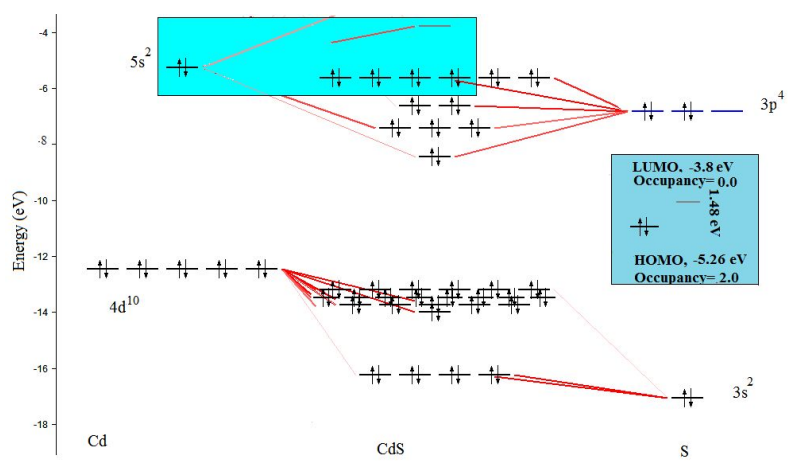

Fig. 2. The MOT structure for a $\mathrm{CdS}$ cluster in the ground state with enlarged view of HOMO and LUMO in colored box.

The atoms in metals are in the form of cations due to plenty of free electrons, whereas the atoms in non-metals are in the form of anions. On (a)
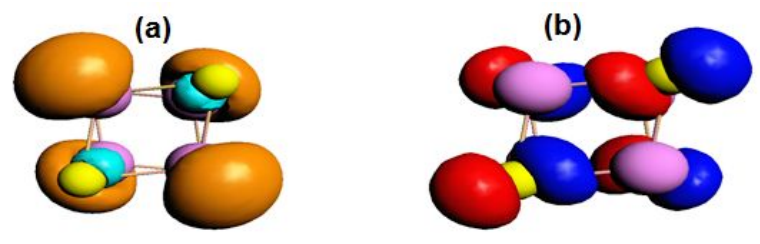

Fig. 3. Isosurfaces plots of (a) HOMO (b) LUMO for CdS cluster shown in Fig. 1.

the other hand, the empty LUMO located at $-3.774614 \mathrm{eV}$ has major contribution from $5 \mathrm{~s}^{2}$ valence electrons of $\mathrm{Cd}$, which is represented by the dark red line and has no contribution from $3 \mathrm{p}^{4}$ substate of S. As shown in Fig. 2, LUMO gives the charge occupation probability in excited state of definitely higher energy than HOMO. In LUMO, which is different from HOMO due to large occupation possibility, a new electron distribution is observed. HOMO and LUMO gap for CdS cluster in the ground state is found equal to $1.48 \mathrm{eV}$, 
Table 3. The bond length, bond angle and bond order for pure and doped CdS.

\begin{tabular}{cccc}
\hline Model & $\begin{array}{c}\text { Bond } \\
\text { length }(\mathrm{pm})\end{array}$ & $\begin{array}{c}\text { Bond } \\
\text { angle (degree) }\end{array}$ & $\begin{array}{c}\text { Bond } \\
\text { order }\end{array}$ \\
\hline \hline CdS cluster & 2.63508 & $180^{\circ}$ & 0.1 \\
Sc-CdS cluster & 275.8 & $180^{\circ}$ & 0.1 \\
\hline
\end{tabular}

which is in agreement with the reported data of energy gap of $1.5 \mathrm{eV}$ calculated for wurtzite $\mathrm{CdS}$ cluster in [32]. The energy level diagram of Sc doped semiconductor nanoparticles is depicted in Fig. 4 with the corresponding HOMO-LUMO location inside the colored box.

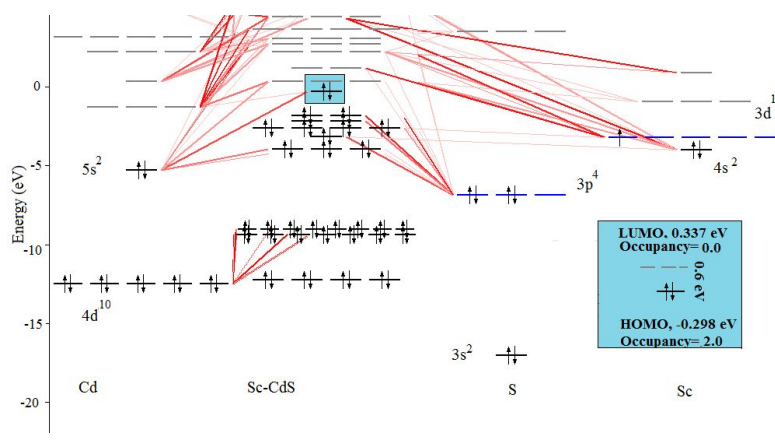

Fig. 4. The MOT structure for Sc doped CdS cluster with enlarged view of HOMO and LUMO in the colored box.

(a)

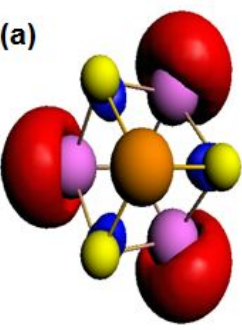

Fig. 5. Isosurfaces plots of (a) HOMO, (b) LUMO for doped $\mathrm{CdS}$ cluster.

The HOMO for Sc doped CdS nanoparticles lies at $-0.298420 \mathrm{eV}$ having occupancy of two electrons with opposite spins and shows a large contribution of electrons from the $5 \mathrm{~s}^{2}$ state of $\mathrm{Cd}$, indicated by the dark red line. Similarly, the LUMO lies at $-0.337615 \mathrm{eV}$ with zero electron occupancy of $5 s^{2}$ state which shows a small contribution from $\mathrm{S}$ and $3 \mathrm{~d}^{1}$ valence electron of Sc, as indicated by the red lines in Fig. 4. It is also evident from the figure that $\mathrm{Sc}$ has unfilled $\mathrm{d}^{1}$ orbital. Moreover the excited $4 s^{2}$ orbital has the same contribution in the core electrons state but no contribution to HOMO. Upon Sc doping of CdS nanoparticles striking changes appear in the HOMO-LUMO gap as shown in the figure. This indicates that the HOMO-LUMO gap decreases from $1.48 \mathrm{eV}$ to $0.63 \mathrm{eV}$. Hence, Sc doping affects the HOMOLUMO gap which may be due to the contribution of $d$ orbital of Sc. The reduction of HOMO-LUMO gap can be explained by sp-d exchange interaction between sp orbital of the base material and localized d orbital of the dopant [33]. This change in energy gap may be useful to design a suitable window material for solar cells. The graphical representation and images of the discussed numerical results for HOMO-LUMO of Sc doped CdS nanoparticles are shown by isosurface plots in Fig. 5a and 5 b. Fig. 5a shows the isosurface plots for a HOMO having the major contribution of spherically symmetric s-orbitals. The molecular orbital lobes of the isosurface plots for LUMO in Fig. 5b also consist of a spherical symmetric s-orbital but a new molecular orbital lobe of d-shape also appears, which clearly indicates the portion of Sc unfilled d-orbital.

The DOS plot for CdS cluster in the ground state, in Fig. 6a shows that there are a number of energy levels available at energy interval between $-16 \mathrm{eV}$ and $25 \mathrm{eV}$. The Fermi energy level lies at $-5 \mathrm{eV}$ below which all states are occupied and above which all states are empty. At $-14 \mathrm{eV}$ a huge collection of electrons is drawn. The DOS for CdS in the ground state is in perfect agreement with its MOT structure shown in Fig. 2. In comparison with the CdS cluster, the density of states for Sc doped $\mathrm{CdS}$ cluster are depicted in Fig. $6 \mathrm{~b}$. 


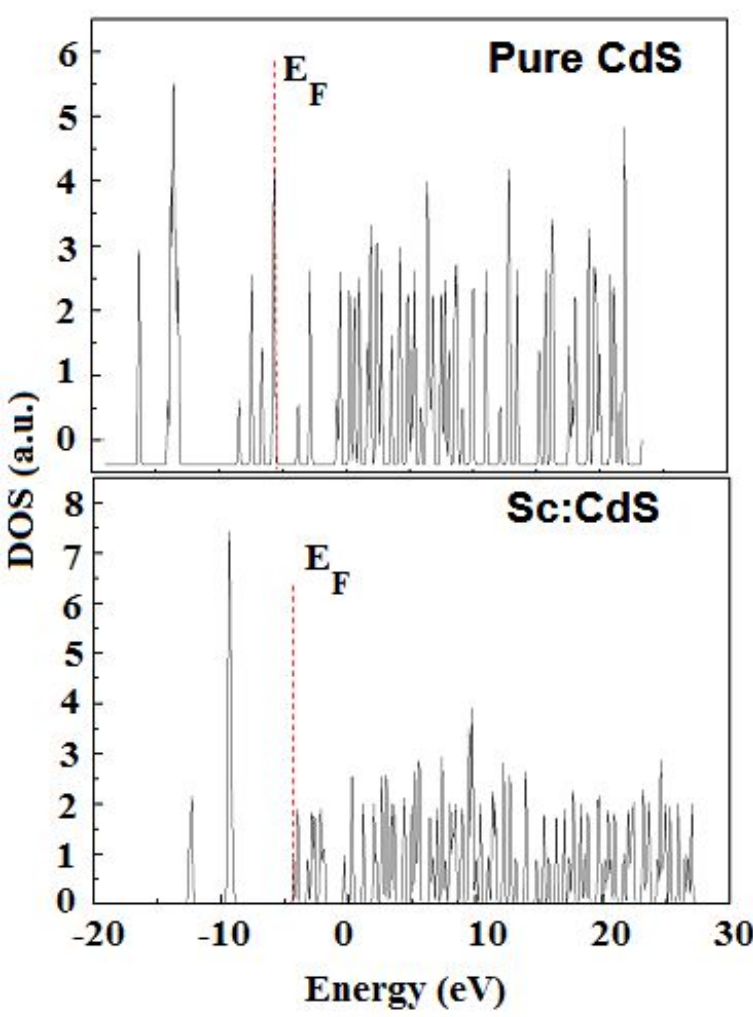

Fig. 6. (a) The DOS plot for pure CdS cluster in the ground state, (b) The DOS plot for Sc doped CdS nanoparticles.

\subsection{Optical properties}

The calculated infrared spectra to study different modes of vibrations of pure and Sc doped $\mathrm{CdS}$ nanoparticles are shown in Fig. 7 in such way that Fig. 7a is related to pure CdS nanoparticles and Fig. $7 \mathrm{~b}$ to Sc doped CdS nanoparticles. The spectrum in Fig. 7a consists of three peaks and each peak represents one mode of vibration; so there are three modes of vibrations in the IR spectrum of pure CdS nanoparticles. These modes of vibrations appear at frequencies $99 \mathrm{~cm}^{-1}, 207 \mathrm{~cm}^{-1}$, $282 \mathrm{~cm}^{-1}$, respectively, as shown in Fig. 7a. The first absorption peak for the CdS cluster at the ground vibrational state lies in far-infrared region and represents lattice vibrational excitation. The lattice vibrations are intermolecular vibrations regarded as inherent molecular property due to regular vibrations of heavy atoms about their mean position, in a synchronized way, so as the atoms have never locked in their positions. The other two modes of vibrations lie in the mid-IR region, which shows that $\mathrm{CdS}$ clusters undergo the fundamental intermolecular vibration. The atoms of $\mathrm{CdS}$ cluster vibrate in three different ways, i.e. symmetric stretching mode, antisymmetric stretching mode and scissoring mode, which were observed by the ADF program. The first peak is due to symmetric stretching mode which shows compactness and inter and intra molecular forces that bind the molecules. The second peak is due to scissoring mode and the last one is due to antisymmetric stretching mode of vibrations of $\mathrm{CdS}$ nanoparticles. The values of characteristic IR-frequencies of different modes of vibrations reported in literature are $242 \mathrm{~cm}^{-1}, 243 \mathrm{~cm}^{-1}$, etc. [34]. It has also been found in the literature that some modes were observed in the range of 85 to $97 \mathrm{~cm}^{-1}$ and 212 to $207 \mathrm{~cm}^{-1}$. Thus, our calculated values of frequency of each mode of vibrations are close to the experimental values.

In the vibrational spectrum of the doped $\mathrm{CdS}$ nanoparticles we have also observed three modes of vibrations which appeared at frequencies $60 \mathrm{~cm}^{-1}, 196 \mathrm{~cm}^{-1}, 358 \mathrm{~cm}^{-1}$, respectively, with slightly different frequencies, shown in Fig. 7b. The appearance of broad bands instead of steep lines in the vibrational spectra manifests the molecular nature of the material. These IR active modes of vibrations also show that Sc doped CdS clusters have highly symmetrical structure. The images of these peaks obtained with the aid of the ADF-GUI program are given below.

The multipeak fitting analysis has shown different available modes of vibrations.

\section{First peak}

The first peak appearing at the lowest frequency of $60 \mathrm{~cm}^{-1}$ in the far-infrared region reveals an IR active mode of vibration, i.e. rocking mode with IR intensity of $3.57 \mathrm{~km} / \mathrm{mol}$. This mode of vibration is appearing due to lattice vibration.

\section{Second peak}

The second broad peak at the frequency of $196 \mathrm{~cm}^{-1}$ lying in the far-IR region reveals an 


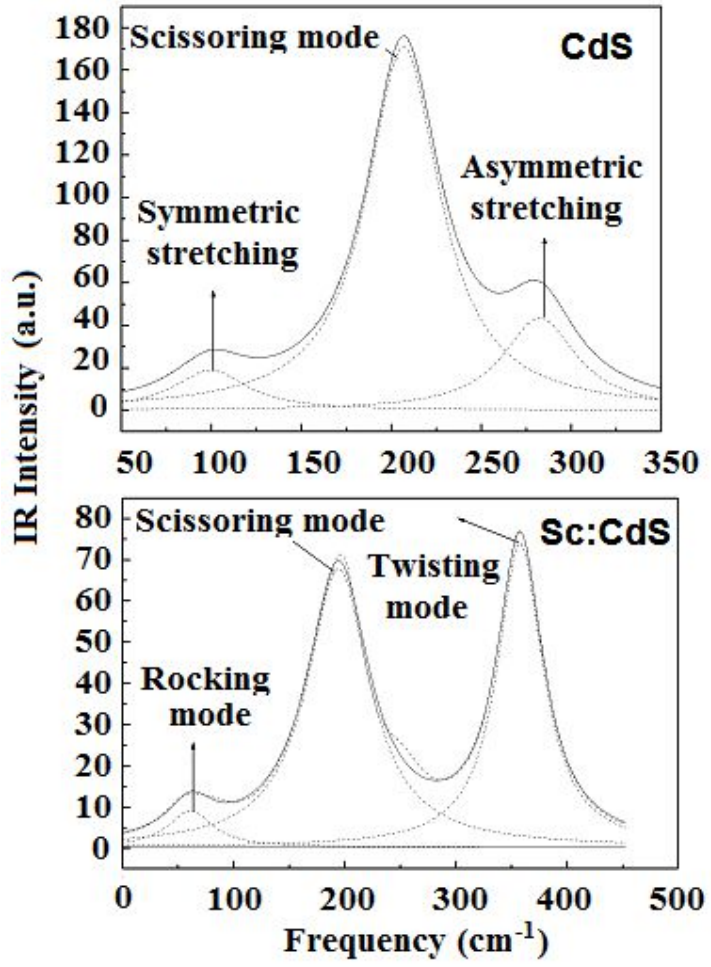

Fig. 7. (a) Calculated IR spectrum of pure $\mathrm{CdS}$ nanoparticles and (b) Sc doped CdS.

IR active mode of vibration, i.e. scissoring mode with intensity of $57 \mathrm{~km} / \mathrm{mol}$.

\section{Third peak}

The third intermediate peak in the absorption band at $356 \mathrm{~cm}^{-1}$ lying in the mid-IR region (200 to $300 \mathrm{~cm}^{-1}$ ) shows an IR active mode, i.e. twisting mode with specific frequency. This mode appears due to intermolecular vibrations.

The Raman spectra for the wurtzite structure of pure cadmium sulphide cluster consisting of 8 atoms and $\mathrm{CdS}$ doped with Sc are shown in Fig. 8. Different peaks observed in the Raman spectrum shown in Fig. 8a point to different Raman modes of CdS nanoparticles. These Raman modes are observed at frequencies $100 \mathrm{~cm}^{-1}, 142 \mathrm{~cm}^{-1}, 195$ $\mathrm{cm}^{-1}$, and $289 \mathrm{~cm}^{-1}$, as labeled in the figure. The first, second and fourth Raman modes represent symmetric stretching modes observed in the ADF graphical user interface program, which is close to the values reported in the literature for the stretching modes of $\mathrm{CdS}$ nanoparticles in the range of 134 to $260 \mathrm{~cm}^{-1}$ [35]. It is also clear that the Raman modes are shifted towards high frequencies, therefore, we can say that a red shift occurs in the Raman spectra of pure CdS nanoparticles because of the coherent anti-Stokes Raman scattering. The third mode represents the bending mode, i.e. twisting, as observed in ADF program.

In comparison with the modes of CdS nanoparticles, the Raman modes of Sc doped CdS nanoparticles show three modes of vibrations in which the middle peak exhibits broadening as compared to other two, as shown in Fig. 8b. These modes of vibrations appear at the frequencies of $60 \mathrm{~cm}^{-1}$, $200 \mathrm{~cm}^{-1}$ and $350 \mathrm{~cm}^{-1}$; each frequency is related to a specific bending or stretching mode of vibration, i.e. symmetric stretching mode, scissoring mode, and wagging mode, respectively, with

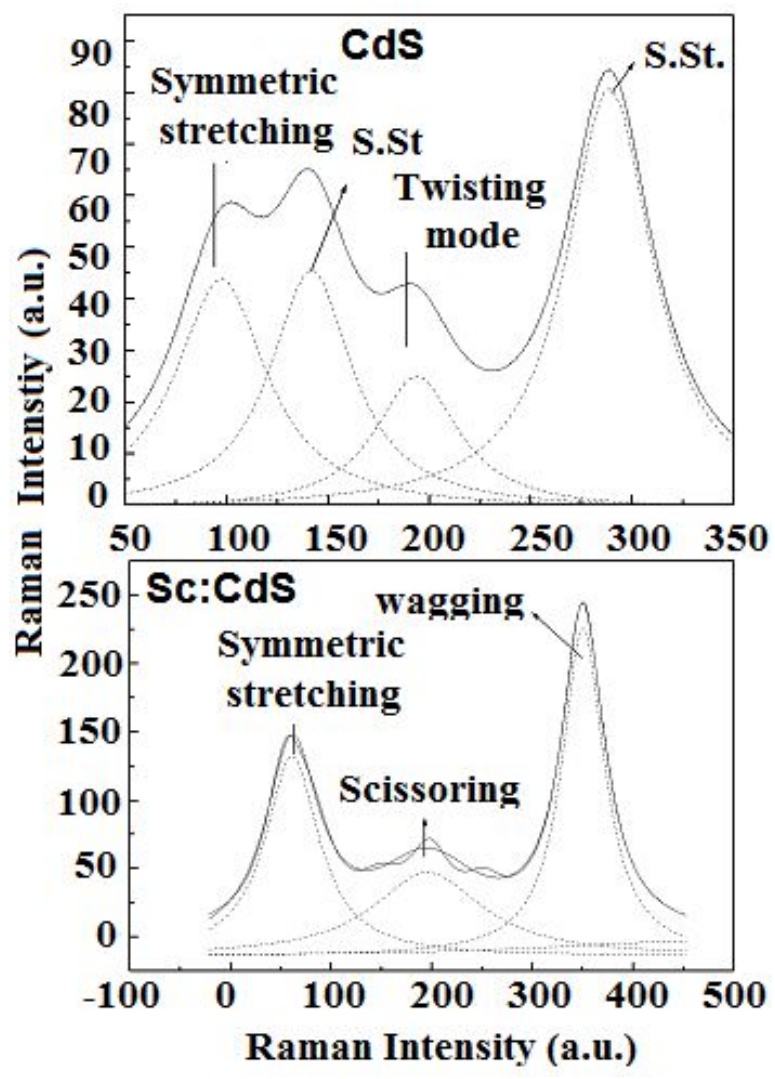

Fig. 8. The calculated Raman spectra (a) of pure CdS nanoparticles and (b) Sc doped CdS. Multifitting analysis shows different stretching and bending modes of vibration. 


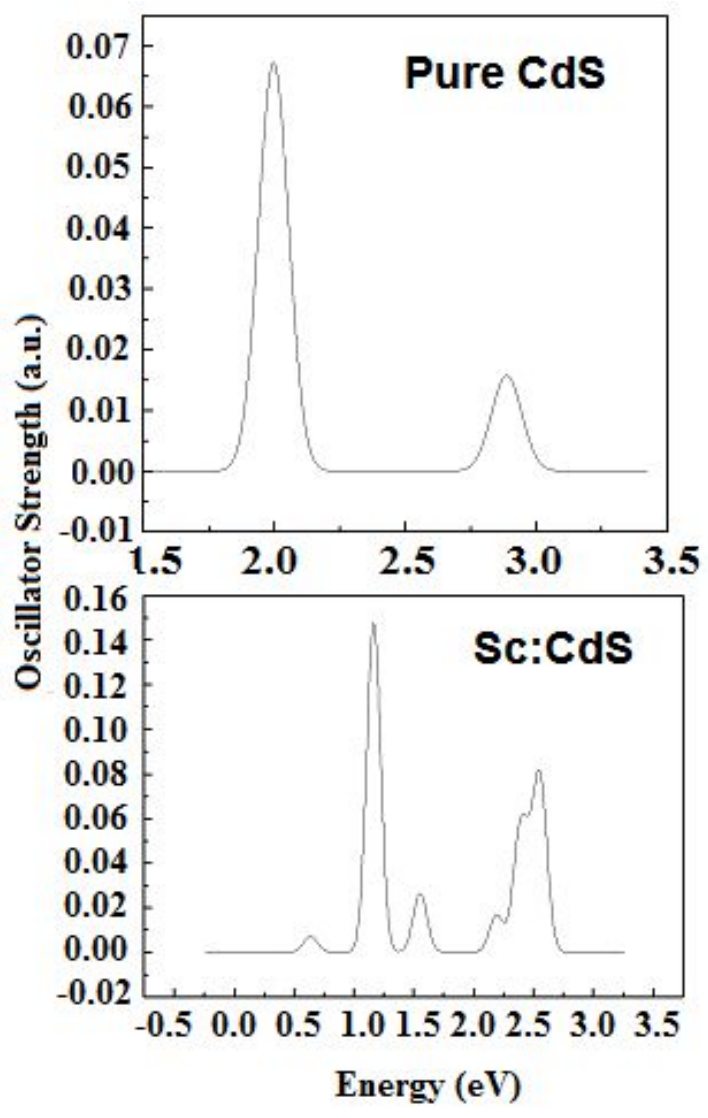

Fig. 9. The calculated excitation spectra (a) for pure $\mathrm{CdS}$ nanoparticles and (b) Sc doped CdS nanoparticles.

the corresponding Raman intensities of 57, 49 and $201 \mathrm{~km} / \mathrm{mol}$, which is clearly seen in the ADF.

The excitation spectra were calculated for pure and Sc doped $\mathrm{CdS}$ nanoparticles using ADF graphical user interface. The most important low lying excited states and high lying states of our pure and doped molecules were compared to the experimental and TDFT results of free-base porphin (FBP) molecule in Table 4 and Table 5. The excitation spectrum consists of many hidden peaks due to excitation between singlet to singlet and singlet to triplet, but due to zero oscillator strength and zero dipole moments these peaks have negligible magnitudes, as noted from ADF-GUI, and do not appear in the absorption spectrum. Since these excitations have specific oscillator strength as well as transition dipole moments, we observe clear bands in the absorption spectrum. Thus, the spectrum of pure CdS cluster shown in Fig. 9a consists of two peaks.

The first peak was assigned to the pair S-S1T1 excitation and the other was assigned to S-S1T2 excitation; their corresponding oscillator strengths and transition diapole moments are also given in Table 4.

The spectrum of Sc doped CdS cluster consists of six peaks and each peak represents an absorption band. These bands are assigned to the excitation from the ground singlet state to different singlet excited states as shown in Table 5. In comparison to pure $\mathrm{CdS}$ cluster the excitation peaks for Sc doped CdS cluster appear at different excitation energies and oscillator strength. From such information we can relate the positions and magnitudes in the absorption spectrum with the geometrical and electronic structure of the molecule.

\section{Conclusions}

It is concluded from the whole discussion that the electronic properties drastically change depending on Sc doping in $\mathrm{CdS}$ nanoparticles. This fact is evident from HOMO-LUMO gap whose value decreases from $1.48 \mathrm{eV}$ to $0.6 \mathrm{eV}$ upon doping. The spectral analysis has revealed different modes of vibrations. The Raman studies revealed that some new modes, such as symmetric stretching mode, scissoring mode and wagging mode appear at frequencies $60 \mathrm{~cm}^{-1}, 200 \mathrm{~cm}^{-1}$, $350 \mathrm{~cm}^{-1}$, respectively, in the doped CdS cluster with the corresponding intensities of 57, 49 and $201 \mathrm{~km} / \mathrm{mol}$. Similarly, in the IR spectrum we have observed three modes of vibrations at frequencies $60 \mathrm{~cm}^{-1}, 196 \mathrm{~cm}^{-1}, 358 \mathrm{~cm}^{-1}$ representing rocking mode, scissoring mode and twisting mode, respectively, with the corresponding intensities of 3.57 and $57 \mathrm{~km} / \mathrm{mol}$, etc. Finally, the excitation spectrum clearly showed the bands for those excitations having oscillator strength and dipole moments. The first band in pure $\mathrm{CdS}$ was assigned to the S-S1T1 excitation and the second was assigned to S-S1T2 excitation. The spectrum of Sc doped CdS cluster consists of six peaks and each peak represents an absorption band. These bands 
Table 4. DFT and experimental excitation energies (eV) and oscillator strength (f) for pure CdS cluster.

\begin{tabular}{cccccccc}
\hline State & $\begin{array}{c}\text { TDFT } \\
\text { excitation } \\
\text { energy (eV) }\end{array}$ & $\begin{array}{c}\text { TDFT } \\
\text { oscillator } \\
\text { strength }\end{array}$ & $\begin{array}{c}\text { Experimental } \\
\text { excitation } \\
\text { energy of } \\
\text { (FBP) } \\
\text { molecule }\end{array}$ & $\begin{array}{c}\text { Experimental } \\
\text { oscillator } \\
\text { strength of } \\
\text { (FBP) } \\
\text { molecule }\end{array}$ & $\begin{array}{c}\text { TDFT } \\
\text { Transition } \\
\text { dipole } \\
\text { moment }\end{array}$ & $\begin{array}{c}\text { TDFT }^{\mathrm{b}} \\
\text { Oscillator } \\
\text { strength (f) } \\
\text { of (FBP) } \\
\text { molecule }\end{array}$ & $\begin{array}{c}\text { TDFT }^{\mathrm{b}} \\
\text { Excitation } \\
\text { energyof } \\
\text { (FBP) } \\
\text { molecule }\end{array}$ \\
\hline \hline S-S1T1 & 1.98335 & 3.61922 & & 0.01 & 1.57568 & 0.01 & 2.16 \\
S-S1T2 & 1.997 & 0.067249 & $1.98-3.33$ & 1.15 & 0.676866 & 0.0005 & 2.29 \\
\hline
\end{tabular}

a [35]

$\mathrm{b}[36]$

Table 5. DFT and experimental excitation energies (eV) and oscillator strength (f) for Sc doped CdS cluster.

\begin{tabular}{cccccccc}
\hline State & $\begin{array}{c}\text { TDFT } \\
\text { excitation } \\
\text { energy } \\
(\mathrm{eV})\end{array}$ & $\begin{array}{c}\text { TDFT } \\
\text { oscillator } \\
\text { strength (f) }\end{array}$ & $\begin{array}{c}\text { Experimental }^{\mathrm{a}} \text { Experimental } \\
\text { excitation } \\
\text { energy (eV) }\end{array}$ & $\begin{array}{c}\text { EFT } \\
\text { oscillator } \\
\text { strength (f) }\end{array}$ & $\begin{array}{c}\text { DFansition } \\
\text { dipole } \\
\text { moment }\end{array}$ & $\begin{array}{c}\text { TDFT }^{\mathrm{b}} \\
\text { excitation } \\
\text { energy (eV) }\end{array}$ & $\begin{array}{c}\text { TDFT } \\
\text { oscillator } \\
\text { strength (f) }\end{array}$ \\
\hline \hline S-S1A1 & 0.639087 & 0.0070823 & $1.98-2.02$ & 0.01 & 2.57621 & $2.16-2.29$ & 0.01 \\
S-S1E & 1.16634 & 0.14776 & $2.33-2.42$ & 0.06 & 1.60765 & 3.01 & $0.0005-0.04$ \\
S-S2E & 1.55533 & 0.026409 & $3.13-3.33$ & 1.15 & -0.58867 & 2.98 & 0.1338 \\
SS2A1 & 2.19425 & 0.0166374 & 4.25 & $<0.1$ & -4.20 & 3.41 & 0.8962 \\
S-S4E & 2.37752 & 0.0303698 & 3.65 & 0.1 & 0.51058 & 3.47 & $0.7293-0.1688$ \\
S-S3A1 & 2.56275 & 0.0699773 & & & 3.863 & $3.77-3.77$ & 0.1272 \\
\hline
\end{tabular}

are assigned to the excitation from the ground singlet state to different singlet excited states. In comparison to pure $\mathrm{CdS}$ cluster the excitation peaks for Sc doped CdS cluster appear at different excitation energies and oscillator strength.

\section{References}

[1] Dong W., Zhu C., Opt. Mater., 22 (2003), 227.

[2] Yoffe A.D., Adv. Phys., 42 (1993), 173.

[3] Majid A., Ahmad R., Nabi A., Shkoor A., HasSAN N., Nanomater. Nanotechno., 2 (2012), 7.

[4] Brus L.E., J. Phys. Chem., 90 (1986), 2555.

[5] Henglein A., Chem. Rev., 89 (1989), 1861.

[6] Weller H., Adv. Mater., 5 (1993), 88.

[7] Alivisatos A.P., Science, 271 (1996), 933.

[8] Hang P., Bester G., Phys. Rev. B, 85 (2012), 41306.

[9] Chamberlain M.P., Giner C.T., Cardona M., Phys. Rev. B, 51 (1995), 1680.

[10] Vasilevskiy M.I., Rolo A.G., Gomes M.J.M., Solid State Commun., 104 (1997), 381.

[11] Kochman, Singh B., IEEE J. Quantum Elec., 39 (2003), 3.

[12] Green M.A., IEEE T. Electron Dev., 46 (1999), 10.

[13] Nabi A., Majid A., J. Korean Phys. Soc., 67 (2015), 1.

[14] Colvin V.L., Schlamp M.C., Alivisatos A.P., Nature, 370 (1994), 354.
[15] Dabbousi B.O., Onitsuka O., Bawendi M.G., Rubner M.F., Appl. Phys. Lett., 66 (1995), 1316.

[16] WANG S., JARRETT B.R., KAUZlarich S.M., LouiE A.Y., J. Am. Chem. Soc., 129 (2007), 3848.

[17] Pradhan N., Battaglia D.M., LiU Y., Peng X., Nano Lett., 7 (2007), 312.

[18] PARK S.Y., Kim P.J., LeE Y.P., Shin S.W., Kim T.H., RHEE J.K., Adv. Mater., 19 (2007), 3496.

[19] Kulkarni J.C., Kazakova O., Holmes J.D., Appl. Phys. A-Mater, 85 (2006), 277.

[20] Mermillod-Blondin A., Bonse J., RosenFELD A., Hertel I.V., MEShChERYAKov Y.P., Bulgakova N.M., Audouard E., Stoian R., Appl. Phys. Lett., 93 (2008), 041911.

[21] Bogle K.A., Ghosh S., Dhole S.D., Bhoraskar V.N., Fu L.-F., Chi M.-F., Browning N.D., KunDaliya D., Das G.P., Ogale S.B., Chem. Mater., 20 (2008), 440.

[22] Kashiwaba Y., Komatsu T., Nishikawa M., IshikaWA Y., SEgaWA K., HAYASI Y., Thin Solid Films, 408 (2002), 43.

[23] Kashiwaba Y., Isojima K., Ohta, Sol. Energ. Mat. Sol. $C, 75$ (2003), 253.

[24] Abe T., Ohashi S.J., Watanabe S., Kashiwaba M., Phys. Status Solidi., 229 (2002), 1015.

[25] Petre D., Pintilie I., Pentia E., Pintilie I., Botila T., Mater. Sci. Eng. B-Adv., 58 (1993), 238.

[26] Kashiwaba Y., Abe H., Kirita H., Ikeda T., Jpn. J. Appl. Phys., 29 (1990), 1733. 
[27] Mohammad S., Otaqsara T., Yousaf M.H., J. Turk. Phys., 35 (2011), 341.

[28] Jindal Z., Verma N.K., Al E., Annu R.M., Annu. Rev. Mater. Sci., 30 (2003), 475.

[29] Hohenberg W., Kohn, Phys. Rev. B, 136 (1964), 864.

[30] Schwarz K., J. Solid State Chem., 176 (2003), 319.

[31] Hehre W.J., RAdom L., Schleyer VON P.R., POPLE J., Ab initio molecular orbital theory, John Wiley \& Sons, New York - Chichester - Brsibane - Toronto Singapore, 1986.

[32] Xu S., Wang C., Cui Y., Int. J. Quantum Chem., 111 (2011), 156.
[33] Kamruzzaman M., Luna T.R., Podder J., Anowar M.G.M., Semicond. Sci. Tech., 27 (2012), 035017.

[34] Tell B., Damen T.C., Porto S.P.S., J. Phys. Rev., 144 (1966), 771.

[35] Gisbergen V., Rosa S.J.A., Ricciardi A., Baerends G., J. Chem. Phys., 111 (1999), 2499.

[36] Edwards L., Dolphin D.H., Gouterman M., Alder, J. Mol. Spectrosc., 16 (1971), 38.

Received 2014-12-31 Accepted 2015-09-11 